\title{
Activity Status and Cardiovascular Diseases: a Cross-sectional Study Based on the Results of Rafsanjan Cohort Study (2020)
}

\author{
Fatemeh Ayoobi 1, 2, Parvin Khalili ${ }^{3}$, Maryam Mohamadi ${ }^{1,4}$, Hamid Hakimi ${ }^{5}$, Carlo La Vecchia ${ }^{6}$, Narjes Soltani ${ }^{1}$, and \\ Ali Esmaeili-nadimi2,*

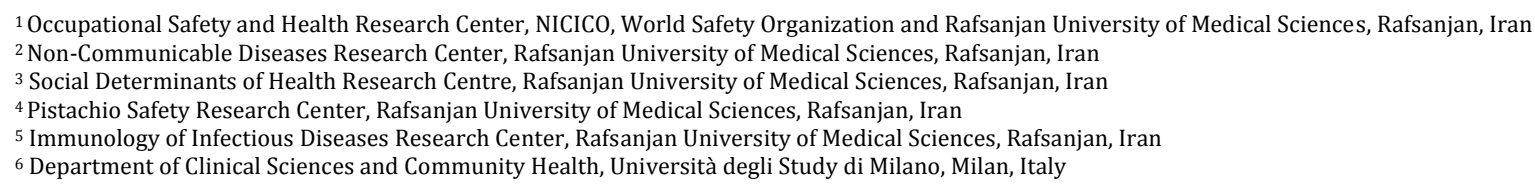

* Corresponding author: Ali Esmaeili-nadimi, Non-Communicable Diseases Research Center, Rafsanjan University of Medical Sciences, Rafsanjan, Iran. Tel: +983434280090; Email: dr_esmaeili_n@yahoo.com

Received 2021 January 09; Revised 2021 February 01; Accepted 2021 March 11.

\begin{abstract}
Background: Cardiovascular disease (CVD) is the leading cause of morbidity and mortality across the globe. Activity status is used as a social class marker of CVDs.

Objectives: The present study aimed to analyze the associations between occupational status and CVDs in Iranian population.

Methods: The present cross-sectional study was conducted on 9,990 subjects aged 35-70 years enrolled in the Rafsanjan Cohort Study (RCS), as one of the Prospective Epidemiological Research Studies in Iran (PERSIAN). Occupational status, socio-demographic characteristics, physical activity, cigarette and hookah smoking, opium use, and alcohol consumption were assessed through six predesigned questionnaires. Anthropometric, body mass index (BMI), medical history, and laboratory tests were also performed. CVDs were defined as the presence of ischemic heart disease (IHD) or myocardial infarction (MI). Prevalence ratios were calculated for each activity status and CVD using Poisson regression models.

Results: The occupational activities were assigned to two classes: homemaker (40.17\%) was the largest group of class I, followed by selfemployed (34.44\%), employed (13.03\%), retired (10.38\%), and unemployed (1.62\%). In class II, the largest group included pistachio farmers $(12.61 \%)$, copper miners $(3.62 \%)$, and others $(83.76 \%)$. A percentage of people were illiterate $(9.50 \%)$, especially in the homemaker group (61.39\%). In general, $8.71 \%$ and $2.98 \%$ of participants suffered from IHD and MI, respectively. After adjusting the socio-demographic and other characteristics, there was no significant association between occupational status and CVDs.

Conclusion: As evidenced by the obtained results, activity status was not associated with the risk of IHD and MI.
\end{abstract}

Keywords: Cardiovascular disease, Ischemic heart disease, Occupational status, Prospective epidemiological research studies in Iran (PERSIAN)

\section{Background}

The estimation of non-communicable diseases (NCDs), especially cardiovascular diseases (CVDs), is of utmost importance in health and policy management (1). Ischemic heart disease (IHD) and stroke are recognized as the first and second causes of death in Iran, respectively. CVDs are the first leading cause of mortality and disabilities, leading to $46 \%$ of all deaths and $20 \%-23 \%$ of the burden of disease in Iran (2).

Although the risk of CVDs in developed countries is higher among people with lower social status (3) in developing populations, they have been reported to be more prevalent in people with upper social status (4). This discrepancy can be partially ascribed to higher exposure to harmful aspects of modernization, such as sedentary lifestyle, cigarette smoking, and obesity in the two mentioned social classes.

The major risk factors of CVD are cigarette smoking, opium use, hypertension, and increased blood lipid level, especially low-density lipoproteins (LDL) cholesterol (5). Other important factors include socio-economic and cultural variants (6), nutrition (7), inadequate physical activity (8), industrialization and urbanization (9), increasing metabolic and physical risk factors (10), as well as low accessibility to primary care and treatment (2). Furthermore, some studies pointed to the association between selected chemicals and CVD risk factors $(11,12)$. Occupational stress is also considered a CVD risk factor (13).

Some studies have presented various occupational activities as a social class marker in the epidemiologic study of CVDs according to their exposure and level of occupational stress $(14,15)$. Occupational status has been reported to be correlated with CVDs or the risk factors of CVD among various populations (16). Among different individual-level factors, aging is considered the strongest predictor of CVDs. The circulatory system 
degenerates with increasing age, as other organs in the body do. Therefore, older individuals are more prone to CVDs, compared to their younger counterparts $(16,17)$. Therefore, health promotion strategies to prevent CVD risk factors, as well as the early detection and treatment of CVDs, can reduce mortality and morbidity worldwide.

Therefore, assiduous attention has now turned to activity status as an explanatory factor to explain CVD risk. Health promotion strategies to prevent and control CVD risk factors, as well as the early detection of the disease and treatment of acute and chronic CVD events, are essential to reduce the burden of CVD in Iran.

\section{Objectives}

The present study aimed to analyze cardiovascular disease risk factors and related health behaviors by activity status.

\section{Methods}

\subsection{Study population}

The study population consisted of 9,990 subjects aged 35-70 years enrolled in the Rafsanjan Cohort Study (RCS), as part of the prospective epidemiological research studies in IrAN (PERSIAN) (18), both urban and suburban areas of Rafsanjan located in South of Iran. Their selection was carried out according to the following criteria: 1) inclusion of areas with minimum migration rates in order to limit loss to follow-up rate, 2) inclusion of populations with different socioeconomic levels, as well as environmental and occupational exposures (18). The study protocol was designed according to the Persian cohort study and was approved by the Ethics Committee of Rafsanjan University of Medical Sciences (IR.RUMS.REC.1399.036).

\subsection{Outcome assessment}

All participants were interviewed by expert interviewers to complete the related questionnaires on demography, socioeconomic status, occupational status, personal habits, biochemical tests, history of the disease, blood pressure, body mass index (BMI), and physical activity. All of the questionnaires were validated in the Persian cohort study (19).

The prevalence of CVD was assessed using selfreported information retrieved from the medical history questionnaire. Prevalent CVDs were IHD and myocardial infarction (MI) based on the selfreporting of the participants. The criteria to diagnose the mentioned disorders were based on a history of angina, MI, coronary bypass surgery, balloon angioplasty, or stent placement in coronary arteries (18).

Education level was originally coded as 1) no schooling, 2) 1-5 years of schooling, 3) 6-12 years of schooling, and 4) university/college degree (18). The daily physical activity of the participants in both leisure and activity status was assessed using a standardized physical activity questionnaire. Each activity was also weighted by its relative metabolic cost, referred to as a metabolic equivalent (MET), thereby deriving MET-hours per day for $24 \mathrm{~h}$ $(19,20)$. The personal habit data, including alcohol consumption, cigarette and hookah smoking, and drug use (e.g., opium, heroin, cocaine, crack, and crystal) were expressed as yes (formerly or currently) and no (never) (18).

The wealth score index (WSI) was categorized into four groups: low income (1st quartile: $\leq$ 0.6069 ), low-middle income (2nd quartile: -0.607 to 0.0349 ), middle-high income (3rd quartile: 0.035 to 1.169) and high income (4th quartile: $\geq 1.170$ ) (19). Fasting serum total cholesterol, high-density lipoprotein cholesterol (HDL cholesterol), lowdensity lipoprotein cholesterol (LDL cholesterol), S.G.O.T (AST), S.G.P.T (ALT), alkaline phosphatase, and triglycerides were measured using a CPA LS analyzer (Coultronics, Margency, France) at the central laboratory in cohort center (18).

The prevalence of hypertension and diabetes mellitus was assessed through a self-reported questionnaire about medical history or drugs. The BMI was also expressed as Mean \pm standard deviation (18). The occupational activity was coded according to the system of the Rafsanjan Cohort Profile. Following that, the obtained groups were assigned to two classes. Class I, including 1) homemaker, 2) unemployed, 3) retired, 4) selfemployed, 5) employed, and class II, including 1) pistachio farmers, 2) copper miners, and 3) others. Subjects in class II may overlap with class I, and some cases had two occupations (18).

\subsection{Statistical analyses}

The prevalence of occupational class, IHD, and MI were compared across categorical and continuous variables using the Pearson $\chi 2$ test, independent t-test, and analysis of variance, respectively. Logistic regression models were employed to investigate the relationships of occupational classes with IHD and MI prevalence. Confounders were identified using a directed acyclic graph based on subject knowledge and the relevant epidemiological literature. Thereafter, they were sequentially entered into models according to their hypothesized strengths of association with occupational class, IHD, and MI. Model 1 included basic demographic characteristics (age, gender, education and WSI) considered to be most strongly related to both exposure and CVDs. Model 2 contained additional adjustments for physical activity level and habit factors (e.g., hookah smoking, alcohol drinking, and misuse/abuse of 
controlled drugs) that were additionally considered to confound occupational IHD and MI associations. Model 3 included all variables in adjusted model 2 and additionally included hypertension, cholesterol, BMI, diabetes mellitus, triglycerides, LDL, HDL, AST, ALT, and alkaline phosphatase. These were hypothesized to be potential confounders on the causal pathways that could explain occupational IHD and MI relationships. In all models, variables of age, cholesterol, BMI, triglycerides, LDL, HDL, AST, ALT, and alkaline phosphatase were entered continuously. Data analyses were performed in STATA software (version 12) (STATA Corp, College Station, TX). All p-values were two-sided, and pvalues $<0.05$ and $95 \%$ confidence intervals were considered statistically significant.

\section{Results}

The number of participants was estimated at 9,990 cases, including 4,655 (46.60\%) males and $5,335(53.40 \%)$ females, according to the baseline phase of the Rafsanjan adult cohort study. Table 1 displays the demographic, lifestyle, personal habits, anthropometric measures, clinical risk factors, and laboratory assessment of participants.

Homemaker $(40.17 \%)$ was the largest group of class I, followed by self-employed (34.44\%), employed (13.03\%), retired (10.38\%), and unemployed $(1.62 \%)$. Class II included pistachio farmers $(12.61 \%)$, copper miners $(3.62 \%)$, and others (83.76\%). In terms of education, a percentage of people were illiterate (9.50\%), especially in the homemaker group (61.39\%).

Regarding physical activity, unemployed

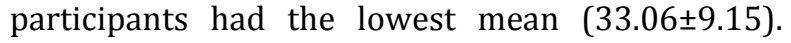
According to the data, the participants were assigned to four categories based on wealth information involving 2342 (23.47\%) =low income, $2863(28.70 \%)=$ low-middle income, 3997 $(40.06 \%)=$ middle-high income, and $775(7.77 \%)=$ high income. The largest proportions of high and low-income subjects were self-employed and homemaker, respectively. The number of people who used cigarettes was calculated at 2,542 (25.4\%), alcohol=1350 (13.6\%), hookah=1,702 (17.2\%), and drug=2,378 (23.8\%). The highest prevalence of the above-mentioned lifestyle habits was observed in the self-employed group.

The proportions of subjects with high blood pressure and diabetes mellitus were $22.5 \%$ and $19.5 \%$, respectively. The highest percentages of mentioned disorders were found among the retired $(31.3 \%$ and $27.7 \%)$. The highest mean scores of cholesterol $(203.79 \pm 47.25)$ and LDL cholesterol (110.69 \pm 31.41$)$ were found among the homemakers. The highest mean scores of triglycerides $(175.66 \pm 128.96)$ and ALT $(25.45 \pm 16.63)$ were reported among the employed. The lowest mean score of HDL cholesterol was reported in the unemployed (55.15 \pm 9.93$)$. The mean BMI was considerably higher in homemakers (29.53 \pm 4.96$)$.

Furthermore, about occupational class II, the highest percentage of diabetes mellitus (20.7\%) and the highest mean of triglycerides (178.55 \pm 119.59$)$ were found in pistachio farmers. The lowest and highest mean scores of HDL cholesterol (53.68 \pm 9.26$)$ and ALT (24.35 \pm 15.64$)$ were reported in copper miners, respectively (Table 1 ).

The prevalence of IHD and MI were obtained at $8.71 \%$ and $2.98 \%$ among all participants (Table 2) and was considerably higher among males. There was a high prevalence of IHD and MI among homemakers and self-employed, respectively. Both of these CVDs were more prevalent among cases with 6-12 years of schooling. Moreover, cholesterol, LDL, HDL, Alkaline phosphate, diabetes mellitus, hypertension, drug use, smokers, and BMI has a direct association with the CVDs, while alcohol consumption and wealth were influential in MI and IHD, respectively (Table 2).

\begin{tabular}{|c|c|c|c|c|c|c|c|c|c|c|c|}
\hline \multicolumn{8}{|c|}{ occupational classes 1} & \multicolumn{4}{|c|}{ occupational classes $2^{*}$} \\
\hline Characteristics & All & Homemaker & unemployed & Retired & Self-employed & Employed & $\begin{array}{c}\text { P- } \\
\text { Value }\end{array}$ & $\begin{array}{l}\text { Pistachio } \\
\text { farmer }\end{array}$ & ¿opper miners & Others & P-Value \\
\hline \multicolumn{12}{|l|}{$\overline{\text { Age( year) }}$} \\
\hline Mean \pm SD & $49.91 \pm 9.57$ & $50.34 \pm 9.50$ & $52.41 \pm 10.73$ & $58.70 \pm 6.00$ & $48.57 \pm 9.32$ & $44.56 \pm 7.23$ & $<0.001$ & $53.92 \pm 9.30$ & $51.15 \pm 10.46$ & $49.26 \pm 9.41$ & $<0.001$ \\
\hline \multicolumn{12}{|l|}{ Gender: no. (\%) } \\
\hline Female & $5335(53.4)$ & 3842 (95.7) & $38(25)$ & $298(27.6)$ & $770(22.4)$ & $387(29.7)$ & \multirow[t]{2}{*}{$<0.001$} & $104(8.3)$ & $21(5.8)$ & $5210(62.3)$ & \multirow{2}{*}{$<0.001$} \\
\hline Male & $4654(46.6)$ & $1711(4.3)$ & $114(75)$ & $783(72.4)$ & $2671(77.6)$ & $915(70.3)$ & & $1156(91.7)$ & $341(94.2)$ & $3158(37.7)$ & \\
\hline \multicolumn{12}{|c|}{ Education-no. (\%) } \\
\hline No schooling & $948(9.5)$ & $582(14.5)$ & $21(14.5)$ & $72(6.7)$ & $253(7.4)$ & $20(1.5)$ & \multirow{4}{*}{$<0.001$} & $144(11.5)$ & $14(3.9)$ & $790(9.5)$ & \multirow{4}{*}{$<0.001$} \\
\hline 1-5 years of school & $2547(25.5)$ & $1452(36.2)$ & $40(17.6)$ & $177(16.4)$ & $799(23.2)$ & $79(6.1)$ & & $306(24.3)$ & $65(18)$ & $2176(26)$ & \\
\hline $\begin{array}{l}\text { 6-12 years of } \\
\text { school }\end{array}$ & 4847 (48.6) & $1752(43.7)$ & $70(48.3)$ & $514(47.5)$ & 2026 (58.9) & $485(37.3)$ & & $624(49.6)$ & $192(53)$ & $4031(48.2)$ & \\
\hline University/college & $1639(16.4)$ & 227 (5.7) & $14(9.7)$ & $318(29.4)$ & $363(10.5)$ & 717 (55.1) & & $183(14.6)$ & $91(25.1)$ & $1365(16.3)$ & \\
\hline \multicolumn{12}{|c|}{ Physical activity. MET } \\
\hline Mean \pm SD & $38.77 \pm 6.36$ & $37.60 \pm 3.47$ & $33.06 \pm 9.15$ & $36.41 \pm 4.46$ & $41.30 \pm 8.34$ & $38.29 \pm 5.78$ & $<0.001$ & $41.63 \pm 9.02$ & $37.67 \pm 7.07$ & $38.39 \pm 5.70$ & $<0.001$ \\
\hline \multicolumn{12}{|c|}{ Wealth score index-no. (\%) } \\
\hline Low income & $2342(23.47)$ & $1154(28.78)$ & $70(48.28)$ & $141(13.04)$ & $841(24.45)$ & $136(10.45)$ & \multirow{4}{*}{$<0.001$} & $242(19.25)$ & $36(9.94)$ & $2064(24.69)$ & \multirow{4}{*}{$<0.001$} \\
\hline $\begin{array}{l}\text { Low-middle } \\
\text { income }\end{array}$ & $2863(28.70)$ & 1263(31.50) & $36(24.83)$ & $298(27.57)$ & 1037(30.15) & $229(17.59)$ & & $380(30.23)$ & $94(25.97)$ & $2389(28.58)$ & \\
\hline $\begin{array}{l}\text { Middle-high } \\
\text { income }\end{array}$ & $3997(40.06)$ & 1435(35.79) & $30(20.69)$ & $536(49.58)$ & $1302(37.86)$ & $694(53.30)$ & & $519(41.29)$ & $175(48.34)$ & 3303(39.52) & \\
\hline High income & $775(7.77)$ & $158(3.94)$ & $9(6.21)$ & $106(9.81)$ & $259(7.53)$ & $243(18.66)$ & & $116(9.23)$ & $57(15.75)$ & $602(7.20)$ & \\
\hline
\end{tabular}




\begin{tabular}{|c|c|c|c|c|c|c|c|c|c|c|c|}
\hline \multicolumn{12}{|c|}{ Cigarette smoking no. (\%) } \\
\hline Yes & $2542(25.4)$ & $106(2.6)$ & $92(60.5)$ & $462(42.7)$ & $1521(44.2)$ & $361(27.7)$ & $<0.001$ & $672(53.3)$ & $179(49.4)$ & $1691(20.2)$ & $<0.001$ \\
\hline No & 7447(74.6) & 3903(97.4) & $60(39.5)$ & $619(57.3)$ & $1920(55.8)$ & 941(72.3) & & $588(46.7)$ & 183(50.6) & $6677(79.8)$ & \\
\hline \multicolumn{12}{|c|}{ Hookah smoking - no. (\%) } \\
\hline yes & 1702(17.2) & $317(7.9)$ & $37(25.2)$ & $153(14.2)$ & $877(25.8)$ & $318(24.6)$ & $<0.001$ & $279(22.3)$ & $95(26.5)$ & 1328(16) & $<0.001$ \\
\hline No & $8206(82.8)$ & $3674(92.1)$ & $110(74.8)$ & $921(85.8)$ & $2527(74.2)$ & $974(75.4)$ & & $970(77.7)$ & $264(73.5)$ & $6972(84)$ & \\
\hline \multicolumn{12}{|c|}{ Alcohol consumption- no. (\%) } \\
\hline yes & $1350(13.6)$ & $25(0.6)$ & $47(32.2)$ & $151(14.1)$ & $899(26.4)$ & $228(17.6)$ & $<0.001$ & $305(24.4)$ & $90(25.1)$ & $955(11.5)$ & $<0.001$ \\
\hline no & $8561(86.4)$ & $3967(99.4)$ & $99(67.8)$ & $923(85.9)$ & $2506(73.6)$ & $1066(82.4)$ & & $944(75.6)$ & $269(74.9)$ & $7348(88.5)$ & \\
\hline \multicolumn{12}{|c|}{ Drug consumption: n (\%) } \\
\hline yes & $2378(23.8)$ & $187(4.7)$ & $89(58.6)$ & $413(38.2)$ & $1406(40.9)$ & $283(21.7)$ & $<0.001$ & $602(47.8)$ & $166(45.9)$ & $1610(19.2)$ & $<0.001$ \\
\hline No & $7611(76.2)$ & $3826(95.3)$ & $63(41.4)$ & $668(61.8)$ & $2035(59.1)$ & $1019(78.3)$ & & $657(52.2)$ & $196(54.1)$ & $6758(80.8)$ & \\
\hline \multicolumn{12}{|c|}{ Hypertension- no. (\%) } \\
\hline yes & $2235(22.5)$ & $1219(30.5)$ & $29(19.5)$ & $337(31.3)$ & $508(14.9)$ & $142(11)$ & $<0.001$ & $258(20.6)$ & $55(15.2)$ & $1922(23.1)$ & $<0.001$ \\
\hline No & $7698(77.5)$ & $2777(69.5)$ & $120(80.5)$ & $740(68.7)$ & $2909(85.1)$ & $1152(89)$ & & $996(79.4)$ & $306(84.8)$ & $6396(76.9)$ & \\
\hline \multicolumn{12}{|c|}{ Diabetes mellitus- no. (\%) } \\
\hline yes & $1933(19.5)$ & $986(24.7)$ & $23(15.4)$ & $298(27.7)$ & $486(14.2)$ & $140(10.8)$ & $<0.001$ & $259(20.7)$ & $53(14.7)$ & $1621(19.5)$ & 0.041 \\
\hline No & $8000(80.5)$ & $3010(75.3)$ & $126(84.6)$ & $779(72.3)$ & 2931(85.8) & $1154(89.2)$ & & $995(79.3)$ & $308(85.3)$ & $6697(80.5)$ & \\
\hline \multicolumn{12}{|c|}{ Diabetes mellitus- no. (\%) } \\
\hline yes & 1933(19.5) & $986(24.7)$ & $23(15.4)$ & $298(27.7)$ & $486(14.2)$ & $140(10.8)$ & $<0.001$ & $259(20.7)$ & $53(14.7)$ & $1621(19.5)$ & 0.041 \\
\hline No & $8000(80.5)$ & $3010(75.3)$ & $126(84.6)$ & $779(72.3)$ & 2931(85.8) & $1154(89.2)$ & & $995(79.3)$ & $308(85.3)$ & $6697(80.5)$ & \\
\hline \multicolumn{12}{|c|}{ Diabetes mellitus- no. (\%) } \\
\hline yes & 1933(19.5) & $986(24.7)$ & $23(15.4)$ & $298(27.7)$ & $486(14.2)$ & $140(10.8)$ & $<0.001$ & $259(20.7)$ & $53(14.7)$ & $1621(19.5)$ & 0.041 \\
\hline No & $8000(80.5)$ & $3010(75.3)$ & $126(84.6)$ & $779(72.3)$ & 2931(85.8) & $1154(89.2)$ & & $995(79.3)$ & $308(85.3)$ & $6697(80.5)$ & \\
\hline \multicolumn{12}{|l|}{ Cholesterol } \\
\hline \multicolumn{12}{|l|}{ Triglycerides } \\
\hline Mean \pm SD & $168.88 \pm 109.22$ & $162.88 \pm 95.77$ & $148.41 \pm 70.81$ & $172.43 \pm 113.7$ & $173.10 \pm 115.19$ & $175.66 \pm 128.96$ & 0.001 & $178.55 \pm 119.59$ & $177.78 \pm 148.42$ & $167.04 \pm 105.43$ & 0.001 \\
\hline \multicolumn{12}{|c|}{ LDL cholesterol } \\
\hline Mean \pm SD & $108.04 \pm 30.50$ & $110.69 \pm 31.41$ & $105.58 \pm 31.94$ & $104.91 \pm 32.69$ & $106.69 \pm 29.09$ & $106.29 \pm 28.53$ & $<0.001$ & $105.43 \pm 30.08$ & $104.97 \pm 30.12$ & $108.56 \pm 30.55$ & $<0.001$ \\
\hline \multicolumn{12}{|c|}{ HDL cholesterol } \\
\hline Mean \pm SD & $57.90 \pm 12.45$ & $60.94 \pm 13.56$ & $55.15 \pm 9.93$ & $56.34 \pm 12.19$ & $55.74 \pm 11.38$ & $55.82 \pm 9.90$ & $<0.001$ & $55.31 \pm 10.32$ & $53.68 \pm 9.26$ & $58.50 \pm 12.77$ & $<0.001$ \\
\hline \multicolumn{12}{|c|}{ 6 } \\
\hline Mean \pm SD & $19.87 \pm 11.81$ & $18.66 \pm 13.01$ & $19.95 \pm 7.60$ & $20.39 \pm 12.26$ & $20.75 \pm 10.95$ & $20.86 \pm 9.65$ & 0.067 & $20.44 \pm 9.66$ & $20.68 \pm 8.26$ & $19.75 \pm 12.23$ & 0.067 \\
\hline \multicolumn{12}{|c|}{ 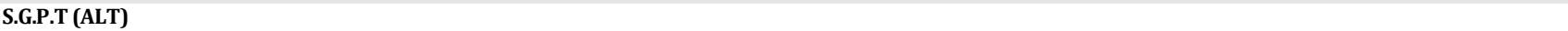 } \\
\hline Mean \pm SD & $21.55 \pm 15.37$ & $18.74 \pm 13.66$ & $19.81 \pm 12.57$ & $21.16 \pm 13.67$ & $23.55 \pm 16.72$ & $25.45 \pm 16.63$ & $<0.001$ & $22.56 \pm 14.18$ & $24.35 \pm 15.64$ & $21.27 \pm 15.52$ & $<0.001$ \\
\hline \multicolumn{12}{|c|}{ Alkaline phosphatase } \\
\hline Mean \pm SD & $225.48 \pm 66.82$ & $224.67 \pm 68.38$ & $235.74 \pm 72.93$ & $230.19 \pm 64.66$ & $226.62 \pm 66.20$ & $219.89 \pm 64.11$ & 0.070 & $227.84 \pm 63.18$ & $231.61 \pm 69.78$ & $224.86 \pm 67.20$ & 0.070 \\
\hline \multicolumn{12}{|c|}{ 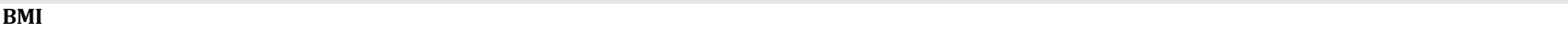 } \\
\hline Mean \pm SD & $27.83 \pm 4.89$ & $29.53 \pm 4.96$ & $25.54 \pm 4.48$ & $26.81 \pm 4.55$ & $26.58 \pm 4.59$ & $26.99 \pm 4.18$ & $<0.001$ & $26.13 \pm 4.33$ & $26.24 \pm 4.00$ & $28.15 \pm 4.94$ & $<0.001$ \\
\hline
\end{tabular}

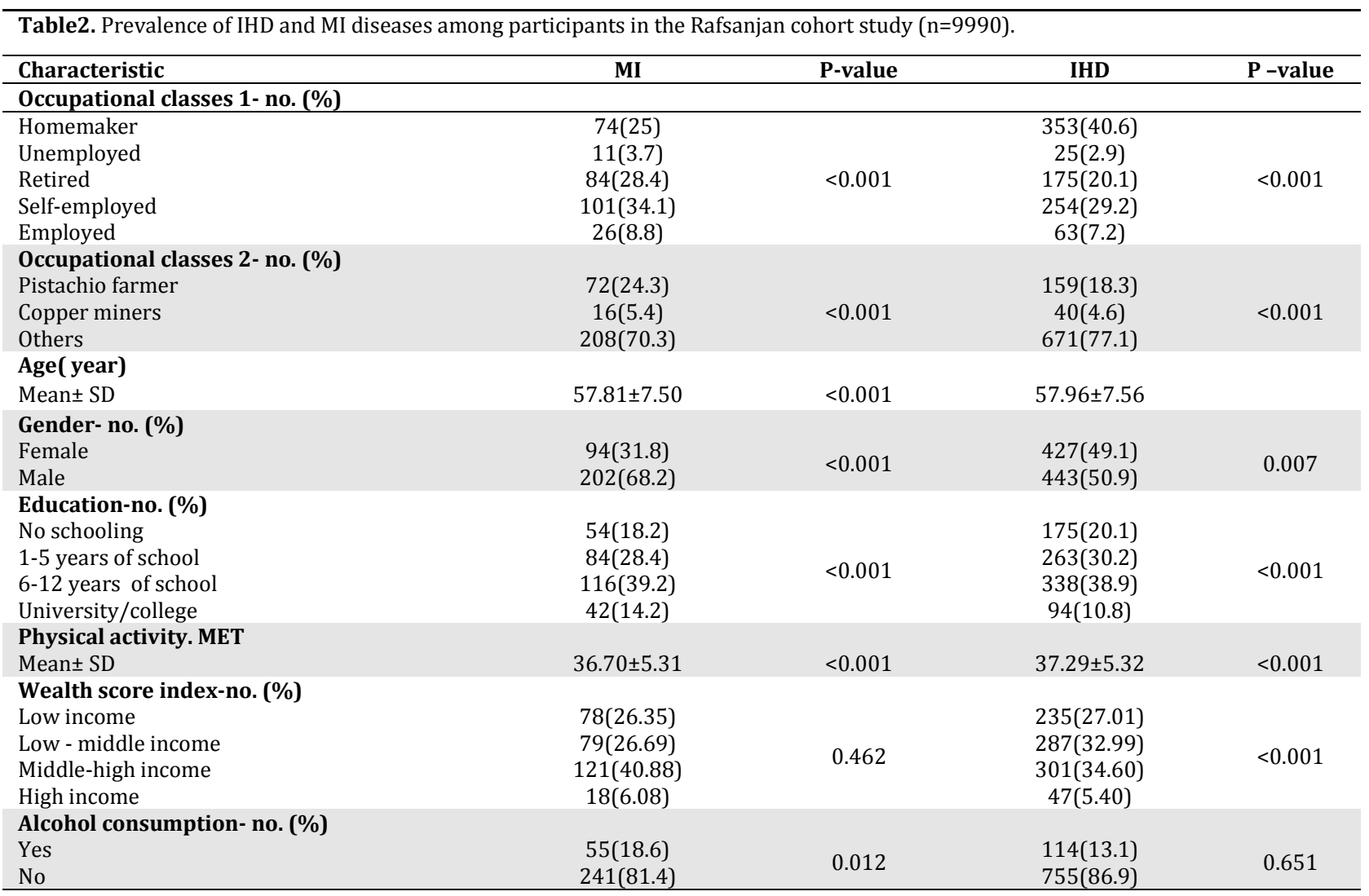




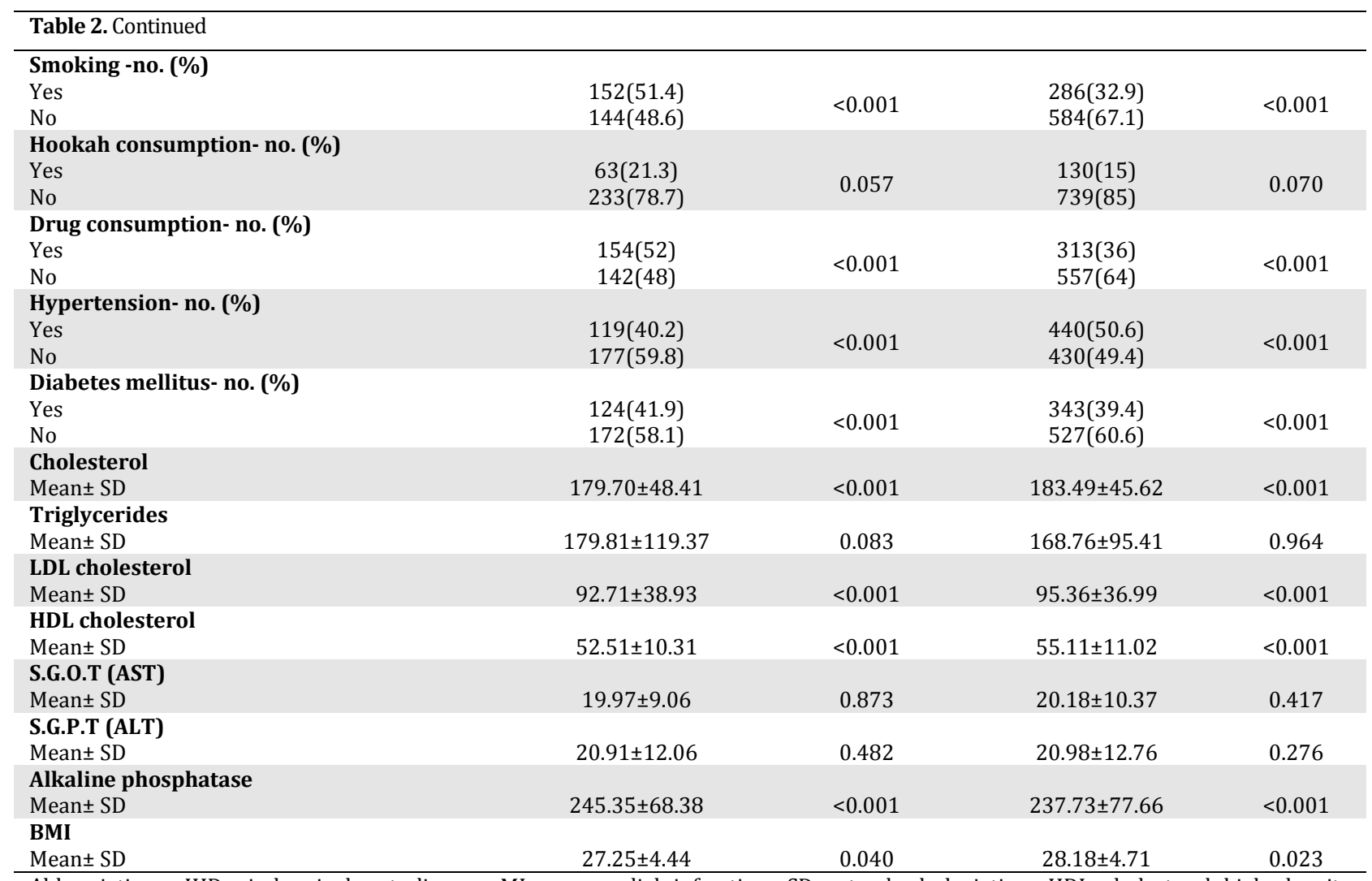

Abbreviations: $\mathrm{IHD}=$ ischemic heart disease; $\mathrm{MI}=$ myocardial infarction; $\mathrm{SD}=$ standard deviation; HDL cholesterol=high density lipoprotein cholesterol; LDL cholesterol= Low density lipoprotein cholesterol; BMI= Body mass index; MET= metabolic equivalent

Table 3 reports the associations of occupational status with IHD and MI using various models.

According to the crude model, the highest odds ratio (OR) for IHD was observed in the unemployed

Table 3 .Associations of activity status with IHD and MI (n=9990)

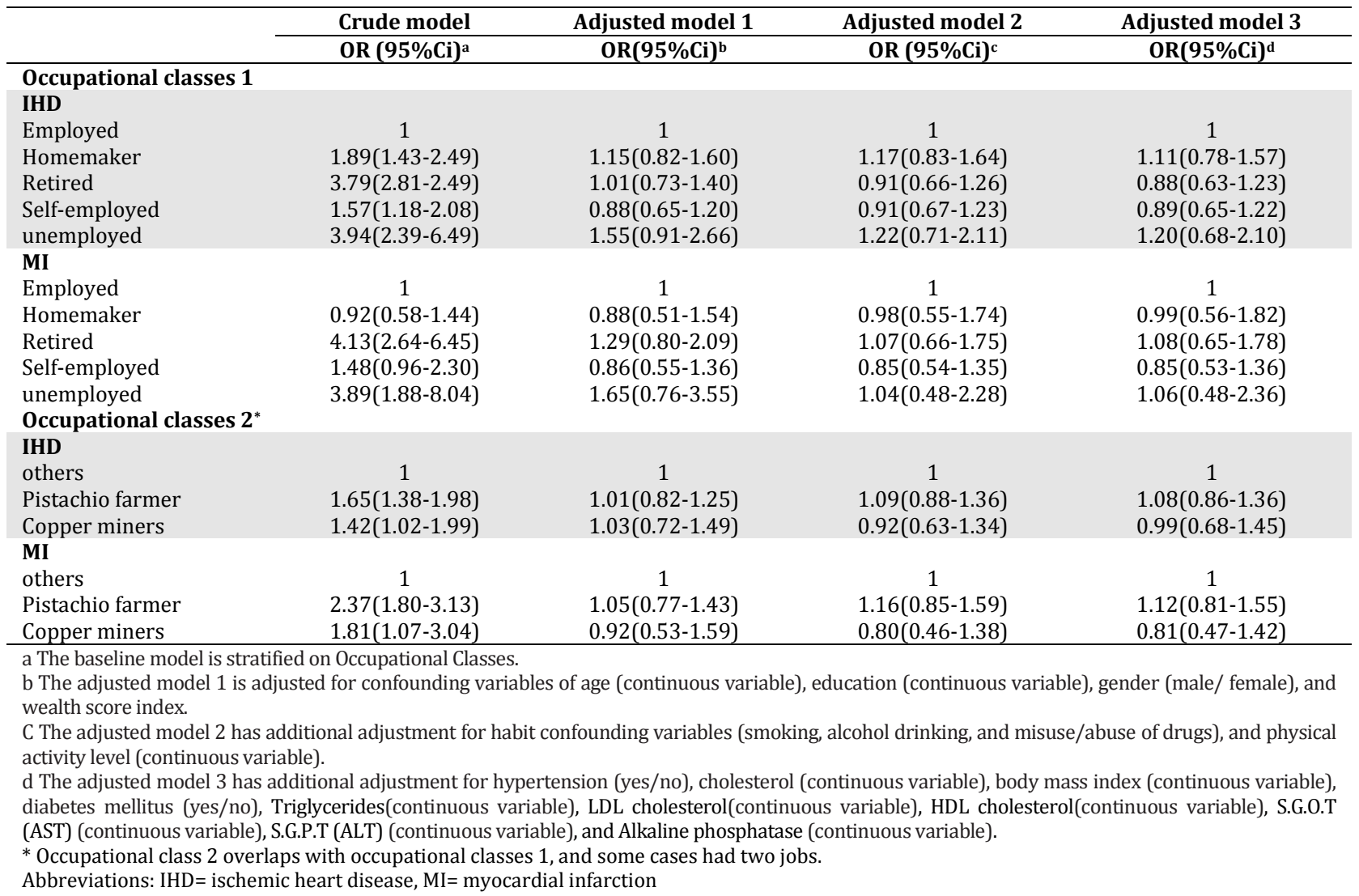


(OR: $3.94,95 \%$ CI 2.39 to 6.49 ), followed by the retired (OR: 3.79, 95\% CI 2.81 to 2.49), compared to homemakers and the self-employed. The OR for MI was significant for the retired (OR: $4.13,95 \% \mathrm{CI} 2.64$ to 6.45), followed by unemployed (OR: 3.89 , 95\% CI 1.88 to 8.04), in occupation class I (Table 3).

In occupation class II, CVDs were frequent in pistachio farmers, firstly followed by copper miners (OR: $1.65,95 \%$ CI 1.38 to 1.98 and OR: $1.42,95 \%$ CI 1.02 to 1.99 for IHD) and (OR: 2.37, 95\% CI 1.80 to 3.13 and OR: $1.81,95 \%$ CI 1.07 to 3.04 for MI), respectively. In model 1 , the effect of age, gender, and wealth score were removed, as well as other confounders in adjustment models 2 and 3. There was no significant association between activity status and CVDs (Table 3).

\section{Discussion}

The present research was conducted based on a cohort study of 9,990 adults enrolled in the Rafsanjan Cohort Study in both urban and suburban of Rafsanjan. The obtained results demonstrated a slight difference between the frequency of IHD and MI in various activity categories. The highest prevalence of IHD and MI was observed among homemakers and selfemployed cases. In addition, CVDs were more prevalent in pistachio farmers, in comparison with copper miners. Nonetheless, after adjustment for some potential confounders, no relationship was detected between CVDs and job type.

Some studies pointed to a relationship between job status/job exposures and CVDs $(12,21)$. Paglione et al. in a cohort study in Rome showed significant differences in mortality by occupational status and type of job in both males and females, and CVD mortality presented higher risks (21). In their cross-sectional study of 566 working women and 561 age-matched housewives, Ainy and Azizi reported that the frequency of cardiovascular risk factors, including abnormal BMI, hypertension, and hyperlipidemia, was significantly higher among housewives, compare to those in working women (22).

Although the role of psychosocial work stress as a risk factor for cardiovascular diseases has been still under debate, work stressors, such as job strain and long working hours, were associated with a moderately elevated risk of incident coronary heart disease and stroke (23). In the same context, Verdejo et al. indicated that drivers, secretaries, or managers with minimum physical activities have a higher prevalence of cardiovascular risk factors (24).

Some other studies pointed to the relationship of pesticide exposure, especially organophosphate, organelle chlorine, and herbicides, with the risk of CVD and coronary heart disease (CHD) (15). Rafsanjan has abundant agricultural lands and about 88,000 hectares of pistachio orchards (18). There is a high level of exposure to pesticides, including organophosphate, organ chlorine, and herbicides in Rafsanjan (25). Therefore, about 700 tons of pesticides are annually used for pistachios in this city. The results of some agricultural studies also suggested that exposure to pesticides is associated with high blood pressure as a risk factor for CVD and other related diseases $(26,27)$. Nevertheless, no relevant association was found in the present study.

$\mathrm{Xu}$ et al. demonstrated that both lifestyle and sociodemographic factors were important correlates of CVDs. Moreover, they found that the incorporation of contextual factors, including environmental hazards and neighborhood characteristics measured at the county level, was useful in determining the individual-level heterogeneities in the incidence of CVD (16).

In all afore-mentioned studies, the relationship between activity status and CVDs was adjusted for cigarette smoking (28), stress $(14,29)$, financial status (6), physical activity (8), education (6), blood pressure (11), and occupational exposures (12). In the current study, no significant relationship was observed between occupational classes and CVDs after allowance for the above-mentioned confounders. Among the notable strengths of the present study, one can refer to its large sample size and the careful allowance for a number of relevant confounders. On the other hand, the limitation of the study included the fact that some individuals who reported agricultural activities (farmers) may have been exposed to selected chemicals or may have used workers to use such chemicals. In any case, we can exclude the major role of such exposures in this dataset.

\section{Conclusion}

The findings of the present study indicated that after the elimination of the effect of confounding factors, there was no direct statistical relationship between activity status and CVDs.

\section{Footnotes}

Author's contributions: F. Ayoobi was involved in designing the study, collecting the data, and drafting the manuscript for content. P. Khalili designed the study, analyzed the data, and drafted the manuscript for content. M. Mohamadi, H. Hakimi, C. Vecchia, and N. Soltani were engaged in designing the study and critically revising the manuscript for important intellectual content. A. Esmaeili-nadimi designed the study, collected the data, and drafted the manuscript for content. All authors have approved the final version of the manuscript and agree to be accountable for all aspects of the 
research.

Conflicts of Interest: The authors declare that they have no conflict of interest regarding the publication of the current study.

Ethical Approval: The study protocol was designed according to the Persian cohort study and was approved by the Ethics Committee of Rafsanjan University of Medical Sciences (Ethical codes: IR.RUMS.REC.1399.036).

Informed Consent: Not applicable

\section{References}

1. Sadeghi M, Haghdoost AA, Bahrampour A, Dehghani M. Modeling the burden of cardiovascular diseases in Iran from 2005 to 2025: the impact of demographic changes. Iran J Public Health. 2017;46(4):506-16. [PubMed: 28540267].

2. Sarrafzadegan N, Mohammadifard N. Cardiovascular disease in iran in the last 40 years: prevalence, mortality, morbidity, challenges and strategies for cardiovascular prevention. Arch Iran Med.2019;22(4):204-10. [PubMed: 31126179].

3. Movsisyan NK, Vinciguerra M, Medina-Inojosa JR, LopezJimenez F. Cardiovascular diseases in central and eastern Europe: a call for more surveillance and evidence-based health promotion. Ann Global Health. 2020;86(1):21. doi: 10.5334/aogh.2713. [PubMed: 32166066].

4. Suglia SF, Campo RA, Brown AG, Stoney C, Boyce CA, Appleton AA, et al. Social determinants of cardiovascular health: Early life adversity as a contributor to disparities in cardiovascular diseases. J Pediatr. 2020;219:267-73. doi: 10.1016/j.jpeds.2019.12.063. [PubMed: 32111376].

5. Akioyamen LE, Genest J, Chu A, Inibhunu H, Ko DT, Tu JV. Risk factors for cardiovascular disease in heterozygous familial hypercholesterolemia: A systematic review and meta-analysis. J Clin Lipidol. 2019;13(1):15-30. doi: 10.1016/j.jacl.2018.10.012. [PubMed: 30527766].

6. Nocon M, Keil T, Willich SN. Education, income, occupational status and health risk behaviour. $J$ Public Health. 2007;15(5):401-5. doi: 10.1007/s10389-007-0120-6.

7. Feng M, Darabi M, Tubeuf E, Canicio A, Lhomme M, Frisdal E, et al. Free cholesterol transfer to high-density lipoprotein (HDL) upon triglyceride lipolysis underlies the U-shape relationship between HDL-cholesterol and cardiovascular disease. Eur J Prev Cardiol. 2020;27(15):1606-16. doi: 10.1177/2047487319894114. [PubMed: 31840535].

8. Wen CP, Wai JPM, Tsai MK, Yang YC, Cheng TYD, Lee MC, et al. Minimum amount of physical activity for reduced mortality and extended life expectancy: a prospective cohort study. Lancet. 2011;378(9798):1244-53. doi: 10.1016/ S0140-6736(11)60749-6. [PubMed: 21846575].

9. Easterlin RA. Industrial revolution and mortality revolution: two of a kind? J Evolut Econ. 1995;5(4):393-408. doi: 10.1007/BF01194368.

10. Sassen B, Cornelissen VA, Kiers H, Wittink H, Kok G, Vanhees $\mathrm{L}$, et al. Physical fitness matters more than physical activity in controlling cardiovascular disease risk factors. Eur J Cardiovasc Prev Rehabil. 2009;16(6):677-83. doi: 10.1097/HJR.0b013e3283312e94. [PubMed: 19734792].

11. Davis-Lameloise N, Philpot B, Janus ED, Versace VL, Laatikainen T, Vartiainen EA, et al. Occupational differences, cardiovascular risk factors and lifestyle habits in South Eastern rural Australia. BMC Public Health. 2013;13(1):1090. doi: 10.1186/1471-2458-13-1090. [PubMed: 24266886].

12. Bulka CM, Daviglus ML, Persky VW, Durazo-Arvizu RA, Lash $\mathrm{JP}$, Elfassy T, et al. Association of occupational exposures with cardiovascular disease among US Hispanics/Latinos. Heart. 2019;105(6):439-48. doi: 10.1136/heartjnl-2018313463. [PubMed: 30538094].

13. Hwang WJ. Cardiovascular disease in Korean blue-collar workers: actual risk, risk perception, and risk reduction behavior. [Doctoral Dissertation]. San Francisco, California: University of California San Francisco; 2010.

14. Frankenhaeuser M, Lundberg U, Fredrikson M, Melin B, Tuomisto M, Myrsten AL, et al. Stress on and off the job as related to sex and occupational status in white-collar workers. J Organ Behav. 1989;10(4):321-46. doi: 10.1002/job.4030100404.

15. Berg ZK, Rodriguez B, Davis J, Katz AR, Cooney RV, Masaki K Association between occupational exposure to pesticides and cardiovascular disease incidence: the Kuakini Honolulu Heart Program. J Am Heart Assoc. 2019;8(19):e012569. doi: 10.1161/JAHA.119.012569. [PubMed: 31550966].

16. Sun W, Gong F, Xu J. Individual and contextual correlates of cardiovascular diseases among adults in the United States: a geospatial and multilevel analysis. Geo J. 2020;85(6):1685700. doi: 10.1007/s10708-019-10049-7.

17. Berry JD, Dyer A, Cai X, Garside DB, Ning H, Thomas A, et al. Lifetime risks of cardiovascular disease. $N$ Engl J Med. 2012;366(4):321-9. doi: 10.1056/NEJMoa1012848. [PubMed: 22276822]

18. Hakimi H, Ahmadi J, Vakilian A, Jamalizadeh A, Kamyab Z, Mehran M, et al. The profile of Rafsanjan cohort study. Eur J Epidemiol. 2020;In Press. doi: 10.1007/s10654-020-006687. [PubMed: 32725579].

19. Eghtesad S, Mohammadi Z, Shayanrad A, Faramarzi E, Joukar F, Hamzeh B, et al. The PERSIAN cohort: providing the evidence needed for healthcare reform. Arch Iran Med. 2017;20(11):691-5. [PubMed: 29480734].

20. Aguilar-Farias N, Brown WJ, Skinner TL, Peeters GG. Metabolic equivalent values of common daily activities in middle-age and older adults in free-living environments: a pilot study. J Phys Act Health. 2019;16(3):222-9. doi: 10.1123/jpah.2016-0400. [PubMed: 30669937].

21. Paglione L, Angelici L, Davoli M, Agabiti N, Cesaroni G. Mortality inequalities by occupational status and type of job in men and women: results from the Rome Longitudinal Study. BMJ Open. 2020;10(6):e033776. doi: 10.1136/bmjopen-2019-033776. [PubMed: 32499259].

22. Kivimäki M, Kawachi I. Work stress as a risk factor for cardiovascular disease. Curr Cardiol Rep. 2015;17(9):630. doi: 10.1007/s11886-015-0630-8. [PubMed: 26238744].

23. Virtanen M, Heikkilä K, Jokela M, Ferrie JE, Batty GD, Vahtera $\mathrm{J}$, et al. Long working hours and coronary heart disease: a systematic review and meta-analysis. Am J Epidemiol. 2012;176(7):586-96. doi: 10.1093/aje/kws139. [PubMed: 22952309].

24. Zimmermann MV, González MG, Galán IL. Cardiovascular risk profiles by occupation in Madrid region, Spain. Rev Esp Salud Publica. 2010;84(3):305-20. doi: 10.1590/s113557272010000300008. [PubMed: 20661528].

25. TavakolianFerdosieyeh V, Karimifared M, Esmaeili A, Hassanshahi G, Vazirinejad R. Pesticide induced complications among pistachio farmers: in the rural area of Rafsanjan, Iran (2010). J Occup Health Epidemiol. 2012; 1(2):111-7. doi: 10.18869/acadpub.johe.1.2.111.

26. Harari R, Julvez J, Murata K, Barr D, Bellinger DC, Debes F, et al. Neurobehavioral deficits and increased blood pressure in school-age children prenatally exposed to pesticides. Environ Health Perspect. 2010;118(6):890-6. doi: 10.1289/ehp. 0901582. [PubMed: 20185383].

27. Andersen HR, Wohlfahrt-Veje C, Dalgård C, Christiansen L, Main KM, Nellemann C, et al. Paraoxonase 1 polymorphism and prenatal pesticide exposure associated with adverse cardiovascular risk profiles at school age. PLoS One. 2012;7(5):e36830. doi: 10.1371/journal.pone.0036830. [PubMed: 22615820].

28. Covey LS, Zang EA, Wynder EL. Cigarette smoking and occupational status: 1977 to 1990. Am J Public Health. 1992;82(9):1230-4. doi: 10.2105/ajph.82.9.1230. [PubMed: 1503163].

29. Burke RJ. Work stress and women's health: occupational status effects. J Busin Ethics. 2002;37(1):91-102. 